\title{
Forschungs-, Poster-, Promotions- und AG-Preise 2019, Sieger Kompetitiver Journal Club
}

\author{
DEUTSCHE \\ GESELLSCHAFT FÜR \\ PATHOLOGIE E.V. \\ Seit 1897 - dem Leben verpflichtet
}

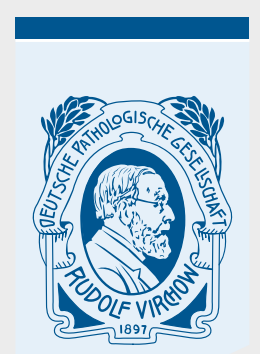

Forschungspreis der DGP (Preisgeld: EUR 1000,00):

Die Axin1-induzierte Hepatokarzinogenese in Mäusen benötigt $\beta$-Catenin aber ist Notch-unabhängig

Frau Dr. med. Katja Evert, Universität Regensburg, Institut für Pathologie, Regensburg

Vier gleichwertige DGP-Posterpreise (Preisgeld: EUR 500,00)

Vergleichende Analyse von zirkulierender und gewebebasierter Tumor-DNA zur molekularen Charakterisierung von Kolonkarzinomen

Frau Anna Haupts, Universitätsmedizin Mainz, Institut für Pathologie, Mainz

Das Urachuskarzinom: Ein Update aktueller molekularer Ergebnisse

Herr PD Dr. med. Henning Reis, Universitätsklinikum Essen, Institut für Pathologie, Essen

Prognostic value of indoleamine 2,3 dioxygenase in patients with higher-risk myelodysplastic syndromes treated with azacytidine

Frau Prof. Dr. med. Martina Rudelius, Ludwig-MaximiliansUniversität München, Pathologisches Institut, München
Combined inactivation of Tp53 and Mir34a enhances invasion and metastasis of colorectal cancer via IL6R/STAT3 and PAI-1 activation

Herr Prof. Dr. med. Heiko Hermeking, Ludwig-MaximiliansUniversität München, Pathologisches Institut, München

Zwei DGP-Promotionspreise (Preisgeld: EUR 750,00)

chREBP ist ein tumorigener Faktor in der hormonell induzierten Hepatokarzinogenese der diabetischen Maus

Herr Vincent Nürnberger, Universitätsmedizin Greifswald, Institut für Pathologie, Greifswald

PRSS23 drives HCC development in context of activated MYC and AKT signaling

Frau Zahra Abadi, Universitätsklinikum Heidelberg, Institut für Pathologie, Heidelberg

Zwei Preise der Arbeitsgemeinschaft Knochen-, Gelenkund Weichgewebspathologie (Preisgeld: EUR 250,00)

FUS-DDIT3 moduliert die transkriptionelle Aktivität von FOXM1 in myxoiden Liposarkomen

Frau Alina Hildebrandt, Universitätsklinikum Münster, Gerhard-Domagk-Institut für Pathologie, Münster

Die miRNA196a-5p reguliert über HOXB7 und BMP4 die WNT1 Expression in Chordomzellen

Frau Carolin Seeling, Universitätsklinikum Ulm, Institut für Pathologie, Ulm

Sieger Kompetitiver Journal Club (Preisgeld für den Sieger EUR 1000.00, Veröffentlichung des Paper-Review in Der Pathologe)

1. Platz: Frau Dr. med. Carina Wenzel, Carl-Gustav-Carus-Universitätsklinikum Dresden, Institut für Pathologie, Dresden

2. Platz: Herr Benjamin Hanke, Otto-von-Guericke-Universität Magdeburg, Institut für Pathologie, Magdeburg

3. Platz: Herr Dr. rer. nat. Marcel Trautmann, Universitätsklinikum Münster, Gerhard-Domagk-Institut für Pathologie, Münster 